\title{
Gestión de la Mesa Ambiental dentro del Proceso de Certificación de Cartagena como Destino Sostenible
}

\author{
Management of the Environmental Table within the Cartagena Certification Process as a \\ Sustainable Destination \\ ${ }^{a}$ Angélica Amaris Severiche ${ }^{2}$ \\ ${ }^{a}$ angelicam22amaris@gmail.com, Semillerista, Estudiante del Programa de Tecnología en Turismo e Idiomas de la Institución \\ Tecnológica Colegio Mayor de Bolívar.
}

Forma de Citar: A. A. Severiche "Gestión de la Mesa Ambiental dentro del Proceso de Certificación de Cartagena como Destino Sostenible”, Rev. Saberes, Vol. 13, No. 02, pp. 19 - 22, 2020.

Recibido: 2/03/2020 Evaluación: 28/05/2020 Aceptado: 30/06/2020 DOI: https://doi.org/10.25213/1794-4384/1302.0002

\section{Resumen}

Cartagena de Indias ha sido de las ciudades pioneras en la implementación y sostenimiento de la certificación de su centro histórico como destino turístico sostenible bajo la norma NTS TS 001 del Ministerio de Comercio, Industria y Turismo. Este logro ha sido posible gracias a la coalición entre diferentes instituciones públicas y privadas y su compromiso con la ciudad. A través de esta investigación se pretende describir la gestión de las organizaciones que conforman la mesa sectorial ambiental, las actividades, metas e indicadores estipulados y alcanzados a lo largo de estos diez años.

\section{Palabras Claves}

Sostenibilidad, centro histórico, Cartagena de Indias, mesa ambiental.

\begin{abstract}
Cartagena de Indias has been one of the pioneer cities in the implementation and sustaining of the certification of its historic center as a sustainable tourist destination according to the NTS TS 001 regulation from the Ministry of Commerce, Industry and
\end{abstract}

Tourism. This achievement has been possible thanks to the alliance between different public and private institutions and their commitment to the city. This research aims to describe the work of the organizations that make up the environmental sector table, the activities, goals, and indicators established and achieved throughout these ten years.

\section{Keywords}

Sustainability, historic center, Cartagena de Indias, environmental table.

\section{Introducción}

Cartagena de Indias al ser un destino turístico sostenible propende por la generación de conocimiento y concienciación en la importancia del uso adecuado, mantenimiento $\mathrm{y}$ preservación del patrimonio natural, histórico y cultural, ofreciendo expresiones culturales y espacios de entretenimiento, comercio y diversión; lo cual afianza la identidad nacional y ofrece oportunidades económicas para los residentes. En Colombia existen 20 destinos turísticos certificados como sostenibles, que deben cumplir con los requerimientos establecidos en la Norma Técnica Sectorial NTS-TS-001, la cual hace énfasis en los requisitos de sostenibilidad que

\footnotetext{
${ }^{2}$ Autor para correspondencia: correo electrónico angelicam22amaris@gmail.com 
debe tener un destino turístico. Cartagena de Indias se diferencia de los demás por ser la primera ciudad colombiana en certificar su Centro Patrimonial como Destino Turístico Sostenible, al recibir por parte del ICONTEC la respectiva acreditación del Centro Histórico, San Diego y Getsemaní.

La entidad encargada de liderar y que tuvo la iniciativa de este proceso es Corpoturismo (Corporación de Turismo Cartagena de Indias) junto con la alianza de otras instituciones públicas y privadas de Cartagena de Indias. Para llevar a cabo el proceso de certificación se crearon en el año 2014, tres mesas de trabajo con base al concepto de sostenibilidad: la mesa sociocultural, económica y la mesa ambiental, cada una de las cuales está compuesta por distintas entidades. Los objetivos planteados de la investigación son fundamentales para plasmar el desarrollo del sistema de gestión de sostenibilidad, los cuales se pretende alcanzar describiendo la gestión del Sistema de Sostenibilidad de Cartagena de Indias en su Centro Histórico, desde la mesa sectorial de trabajo ambiental, evidenciando sus acciones, indicadores e inversión financiera. Identificando las entidades que se han vinculado a la mesa ambiental dentro del proceso de certificación del Centro Histórico desde el año 2009 hasta la fecha como también revisando los logros obtenidos por la mesa ambiental desde los planes de acción. Y, por último, estableciendo el impacto que la gestión de la mesa sectorial de trabajo ambiental ha tenido frente a las problemáticas ambientales en el Centro Histórico.

\section{Metodología}

La investigación es de tipo descriptiva, ya que pretende explicar las características de la problemática identificada, es decir, si en realidad los planes de acción de dichas entidades e inversiones están enfocadas a solucionar las problemáticas ambientales que se presentan en el Centro Histórico de Cartagena de Indias. Como también describir la planificación estratégica, el desarrollo de la normativa que integra transversalmente la certificación en sostenibilidad turística en busca de promover en los turistas y residentes acciones amigables con el ambiente.

Las fuentes primarias se obtendrán de tomas fotográficas del trabajo de campo y entrevistas indagando con las entidades que lideran el proceso de certificación, en cuanto a las secundarias de investigaciones, artículos de periódicos e informes generados por universidades, entidades gubernamentales y ONG's sobre el tema estudiado, en especial, de la normativa en Colombia. Se presenta un análisis de los documentos que permiten servir de base teórica para la orientación de la investigación y a través de la observación se realiza el diagnóstico de la zona que busca responder a la siguiente pregunta: ¿Cuáles son las acciones, indicadores e inversión financiera que están realizando las entidades que conforman la mesa ambiental que contribuyen al proceso de certificación del Centro Patrimonial como Destino Turístico Sostenible?

\section{Discusiones}

El centro histórico como epicentro de las actividades turísticas en la ciudad presenta una serie de problemáticas producto de este ejercicio, tales como: Cajas de registro en mal estado, desborde de agua de alcantarillado, malos olores, pocas canecas de basura lo que provoca que las personas arrojen basura en cualquier lugar, como también la falta de sentido de pertenencia por parte de ellas. Todo esto se ha dado paralelo a los esfuerzos de las autoridades y representantes en desarrollar un destino turístico sostenible a través de la conformación de mesas que se enfocan en los pilares de la sostenibilidad y han desarrollado su actividad por más de 10 años, lo que conduce a la siguiente pregunta: ¿Cuáles son las acciones, indicadores e inversión financiera que están realizando las entidades que conforman la mesa ambiental que contribuyen al proceso de certificación del Centro Patrimonial como Destino Turístico Sostenible?

Justificando la investigación se puede argumentar que la sostenibilidad es un tema 
que está dando mucho de qué hablar, ya que la ONU en 2015 estableció 17 Objetivos de Desarrollo Sostenible (ODS), anteriormente existían los ODM (Objetivos de Desarrollo del Milenio) estaban compuestos de 8 objetivos cuyo vencimiento llegó en el 2015. Cada objetivo tiene metas específicas que deben alcanzarse en los próximos 15 años. Cabe resaltar que el Gobierno colombiano dentro del PND está incorporando y planteando estrategias con los objetivos anteriormente mencionados; lo que ayudará a mejorar el índice de competitividad, ya que, según el Índice Global de Competitividad del Foro Económico Mundial, Colombia cayó 6 puestos en 2018 frente al ejercicio pasado, cuando ocupó el puesto 59, obteniendo así su peor ubicación en los últimos diez años, a pesar de que se perfiló como el tercer país de la región con mayor facilidad para hacer negocios.

Al obtener el Centro Histórico su certificado como Destino Turístico Sostenible contribuye a cumplir las metas de los ODS; ayudando a erradicar la pobreza, promover la prosperidad y el bienestar para todos, proteger el medio ambiente y hacer frente al cambio climático desde las diferentes mesas de trabajos. La mesa ambiental contribuye a ODS número cinco (5): agua limpia y saneamiento, producción y consumo responsable, acción por el clima, vida submarina y vida de ecosistemas terrestres. Es decir, a los números 6,12,13,14 y 15 .

Esto trae como beneficio un mayor de turistas a la ciudad, y a la vez contribuye al dinamismo económico de la misma y al bienestar de la población; ya que la certificación es una garantía de calidad del destino para los turistas y visitantes a Cartagena de Indias.

\section{Resultados Esperados}

De la investigación se espera obtener:

- Directorio de las entidades que han pertenecido a la mesa de ambiental en el proceso de certificación desde el 2009.
- Compendio de las acciones ambientales enmarcadas en el proceso de certificación, desde 2009.

- Documento - informe analítico acerca del impacto que han generado las acciones de la mesa ambiental frente a las problemáticas.

\section{Conclusiones Preliminares}

De la investigación en curso se ha podido concluir:

- La ciudad cuenta con un sistema de sostenibilidad en constante marcha con la participación activa de entidades públicas y privadas, así como los residentes.

- Se han desarrollado 4 planes de acción desde el año 2009, los cuales han sido aprobados por parte de las entidades certificadoras con pocas no conformidades resueltas.

- El sistema ha abarcado gran número de participantes. Se siguen vinculado nuevas entidades.

- El Sistema cuenta con un cronograma de actividades establecido donde se realizan reuniones y socializaciones para alcanzar la meta en común.

- Importante recordar que la construcción en los últimos años ha tenido un desarrollo muy alto en cuanto a la tecnología y diversificación de la misma; tocando el problema que se posee a la hora de diversificación, el cual es no contar con las herramientas para realizar un diseño con el fin de construir con el material deseado. (Granados, 2019).

\section{Referencias Bibliográficas}

Brundtland, G. H. (1987). Nuestro futuro común (Our Common Future). Oxford University Press.

CARTAGENA, R. (13 de agosto de 2016). ElUniversalCtg. Obtenido de https:/www.eluniversal.com.co/cartagena/elcentro-historico-vuelve-recibir-certificaciondel-icontec-232966-JQEU339918

Chavarriaga, W. A. (12 de agosto de 2015). El Heraldo. Obtenido de 
https://www.elheraldo.co/bolivar/centrohistorico-de-cartagena-obtiene-certificadointernacional-de-destino-turistico

City, C. d. (s.f.). Cartagena de Indias World Heritage City. Obtenido de http://www.cartagenadeindias.travel/?la=en\& amp;/corporacion-institucional certificacion$\underline{\text { destino-2 }}$

Gisolf, M. C. (1 de 03 de 2012). Turismo en teoría. Obtenido de https://www.tourismtheories.org/?p=470\&am p;lang=es

Instituto Colombiano de Normas Técnicas y Certificación. (10 de 07 de 2006). FONDO NACIONAL DE TURISMO - FONTUR. Obtenido de FONDO NACIONAL DE TURISMO - FONTUR: https://www.fontur.com.co/aym document/ay $\underline{\mathrm{m} \text { normatividad/2006/NTS TS001 1.pdf }}$

Instituto Colombiano de Normas Técnicas y Certificación. (11 de 12 de 2014). MINCIT. Obtenido de http://www.mincit.gov.co/CMSPages/GetFile aspx?guid=28e59887-a6ce-404a-9675$\underline{848 \mathrm{~d} 52 \mathrm{c} 696 \mathrm{e} 4}$

Ministerio de Comercio, Industria y Turismo. (JULIO de 2019). MINCIT. Obtenido de http://www.mincit.gov.co/CMSPages/GetFile aspx?guid=cf570b29-7f96-40f2-8bb6$\underline{6 \mathrm{f} 75 \mathrm{c} 2653495}$

REPÚBLICA, L. (24 de AGOSTO de 2015). Obtenido de http://www.icontec.org/NC/SP/Artculos\%20e n\%20Prensa/La\%20Rep\%C3\%BAblica\%202 4\%20de \%20agosto $\% 20 \mathrm{de} \% 202015$.pdf

Granados-Páez, M., Bohórquez-Rojas, $R$ y E.J. Barrientos, E. (2019). "Análisis del comportamiento de la guadua "BAMBU", Sostenibilidad, Tecnología y Humanismo, vol. 10 , no. 2 , 49-55. 